\title{
Identification de deux procédés de débitage inédits du bois de cervidés dans les niveaux gravettiens de Laugerie-Haute est et ouest
}

Identification of two unpublished debitage production schemas in the gravettian levels of Laugerie-Haute East and West

\section{Nejma Goutas}

\section{OpenEdition \\ Journals}

Édition électronique

URL : http://journals.openedition.org/paleo/1319

DOI : $10.4000 /$ paleo.1319

ISSN : 2101-0420

Éditeur

SAMRA

Édition imprimée

Date de publication : 1 décembre 2003

Pagination : 255-262

ISSN : 1145-3370

Référence électronique

Nejma Goutas, «Identification de deux procédés de débitage inédits du bois de cervidés dans les niveaux gravettiens de Laugerie-Haute est et ouest », PALEO [En ligne], 15 | 2003, mis en ligne le 04 août 2010, consulté le 07 juillet 2020. URL : http://journals.openedition.org/paleo/1319 ; DOI : https:// doi.org/10.4000/paleo.1319

Ce document a été généré automatiquement le 7 juillet 2020

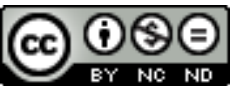

PALEO est mis à disposition selon les termes de la licence Creative Commons Attribution - Pas d'Utilisation Commerciale - Pas de Modification 4.0 International. 


\section{Identification de deux procédés de débitage inédits du bois de cervidés dans les niveaux gravettiens de Laugerie-Haute est et ouest}

Identification of two unpublished debitage production schemas in the gravettian levels of Laugerie-Haute East and West

Nejma Goutas

Cette étude a porté sur le matériel de Laugerie-Haute Est et Ouest appartenant aux faciès récent (Périgordien VI ) et final (Protomagdalénien ou Périgordien VII) du Gravettien, soit 482 pièces conservées au Musée National de Préhistoire aux Eyzies-deTayac et provenant des collections Peyrony et Bordes (tabl. 1).

Tableau 1 - Nombre de pièces étudiées par série, par période et par matière première. Table 1 - Number of studied artefacts by series, by period and by raw material.

\begin{tabular}{|l||l|l|l|l|l|l|l|}
\hline & $\begin{array}{l}\text { Bois de } \\
\text { renne }\end{array}$ & $\begin{array}{l}\text { Bois de } \\
\text { cerf }\end{array}$ & $\begin{array}{l}\text { Bois de cervidé } \\
\text { indéterminé }\end{array}$ & Os & Ivoire & $\begin{array}{l}\text { Matière } \\
\text { indéterminée }\end{array}$ & TOTAL \\
\hline $\begin{array}{l}\text { LHE Gravettien } \\
\text { récent }\end{array}$ & 181 & 5 & 0 & 31 & 18 & 21 & 256 \\
\hline $\begin{array}{l}\text { LHO Gravettien } \\
\text { récent }\end{array}$ & 59 & 1 & 1 & 7 & 3 & 3 & 74 \\
\hline $\begin{array}{l}\text { LHE Gravettien } \\
\text { final }\end{array}$ & 71 & 1 & 1 & 47 & 1 & 31 & 152 \\
\hline TOTAL & 311 & 7 & 2 & 85 & 22 & 55 & 482 \\
\hline
\end{tabular}


2 Le débitage du bois de cervidés à Laugerie-Haute Est et Ouest relève de deux principaux schémas de transformation : par tronçonnage et par extraction. Nous nous attacherons uniquement à décrire et à analyser ce dernier schéma, à travers l'exemple du Gravettien récent de Laugerie-Haute Est, bien que les observations présentées soient également valables pour le Gravettien récent de Laugerie-Haute Ouest ainsi que pour le Gravettien final de Laugerie-Haute Est.

\section{Les matrices de débitage}

3 Celles-ci sont exclusivement en bois de renne, animal dominant dans les restes de faune (Peyrony 1938). Il s'agit à la fois de bois de mue et de bois de massacre. Cette distinction n'est cependant pas possible lorsque les matrices sont localisées sur tronçons de perche ou sur andouillers (tabl. 2).

Tableau 2 - Origine des matrices de débitage. Table 2 - Origin of the debitage matrices.

\begin{tabular}{|l|l|l|l|l|}
\hline Matrice de débitage & Mue & Massacre & Indéterminée & TOTAL \\
\hline bois de renne & 8 & 10 & 18 & 36 \\
\hline bois de cerf & 0 & 0 & 1 & 1 \\
\hline TOTAL & 8 & 10 & 19 & 37 \\
\hline
\end{tabular}

4 Les déchets (épois, chevillure et andouillers) et les matrices de débitage sont abondants ( $\mathrm{n}: 50)$. Ce sont les bois de moyen module qui ont été principalement recherchés comme l'attestent les mesures prises sur les matrices, les déchets de débitage (tabl. 3) mais aussi sur les supports produits et les objets finis. Toutefois, les bois de gros et petit module ont également été exploités mais de façon moindre.

Tableau 3 - Modules des matrices et des déchets de débitage. Table 3 - Modules of matrices and manufacturing wastes.

\begin{tabular}{|l|l|l|l|}
\hline Module & Bois de cerf & Bois de renne & TOTAL \\
\hline Gros & 4 & 6 & 10 \\
\hline Moyen/Gros & 0 & 7 & 7 \\
\hline Moyen & 0 & 23 & 23 \\
\hline Moyen/Petit & 0 & 1 & 1 \\
\hline Petit & 0 & 9 & 9 \\
\hline TOTAL & 4 & 46 & 50 \\
\hline
\end{tabular}


Les matrices de débitage sont fréquemment sur base et perche A (fig. 1), $\mathrm{C}$ ou $\mathrm{BC}$ (plus rarement sur perche $A B C$ ), ainsi que sur andouiller. Un seul épois de cerf a été exploité. Le schéma de transformation par extraction est essentiellement attesté sur bois de renne mais aussi sur quelques rares pièces en bois de cerf. Le débitage de l'os relève quant à lui des schémas de transformation par bipartition et par segmentation ${ }^{1}$. Enfin, ne disposant pour l'ivoire que d'objets finis, nous sommes dans l'impossibilité de reconstituer les procédés employés pour son débitage.

Figure 1 - Nomenclature usuelle des différentes parties du bois de cerf (d'après Billamboz 1979) et du bois de renne (d'après Averbouh 2000).

Figure 1 - Usual nomenclature of the various parts of the deer's (according to Billamboz, 1979) and reindeer's (according to Averbouh, 2000) antlers.

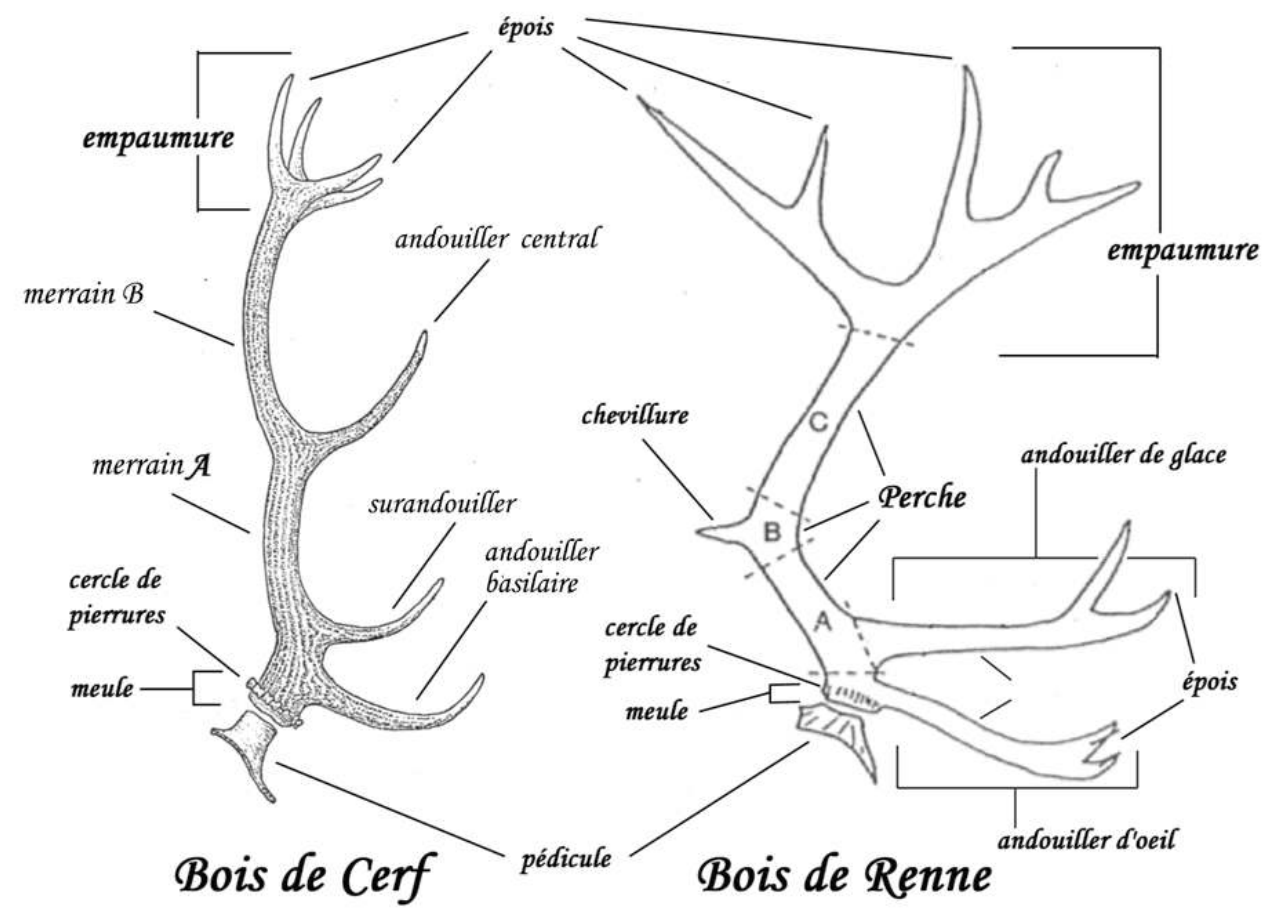

\section{Localisation des extractions sur la ramure}

6 Les matrices présentent généralement une seule extraction et rarement plusieurs. Celles-ci ont majoritairement eu lieu sur les faces postérieure, latéro-postérieure ou antérieure de la perche A. Cette partie de la perche offre en effet, une importante épaisseur de tissu compact et une grande rectitude qui ont, semble-t-il, été recherchées. Le débordement volontaire de certaines extractions sur les faces latérales permettait d'obtenir un support encore plus large. On remarque aussi fréquemment un outrepassé des baguettes, certaines emportant avec elles une partie du cercle de pierrures.

\section{Le schéma de transformation par extraction}

7 La production de baguettes est abondante. Ainsi, plus d'une trentaine d'extractions ont été dénombrées sur les matrices de débitage ainsi que 36 baguettes (brutes de débitage 
ou en cours de façonnage) et plus d'une centaine d'objets réalisés sur ce type de support. Cette production fait intervenir le procédé de double rainurage longitudinal ainsi que deux autres procédés de débitage, inédits jusqu'alors au sein du Gravettien (fig. 2) :

- un procédé de refend reposant sur l'association du tronçonnage et du fendage,

- un procédé mettant en jeu les techniques du rainurage (simple ou double) et du fendage.

Figure 2 - Variabilité du schéma de transformation par extraction dans le Gravettien récent de Laugerie-Haute Est.

Figure 2 - Variability of the transformation schema by extraction for the late Gravettian of Laugerie-Haute Est.

Identification de trois procédés d'extraction de baguette :

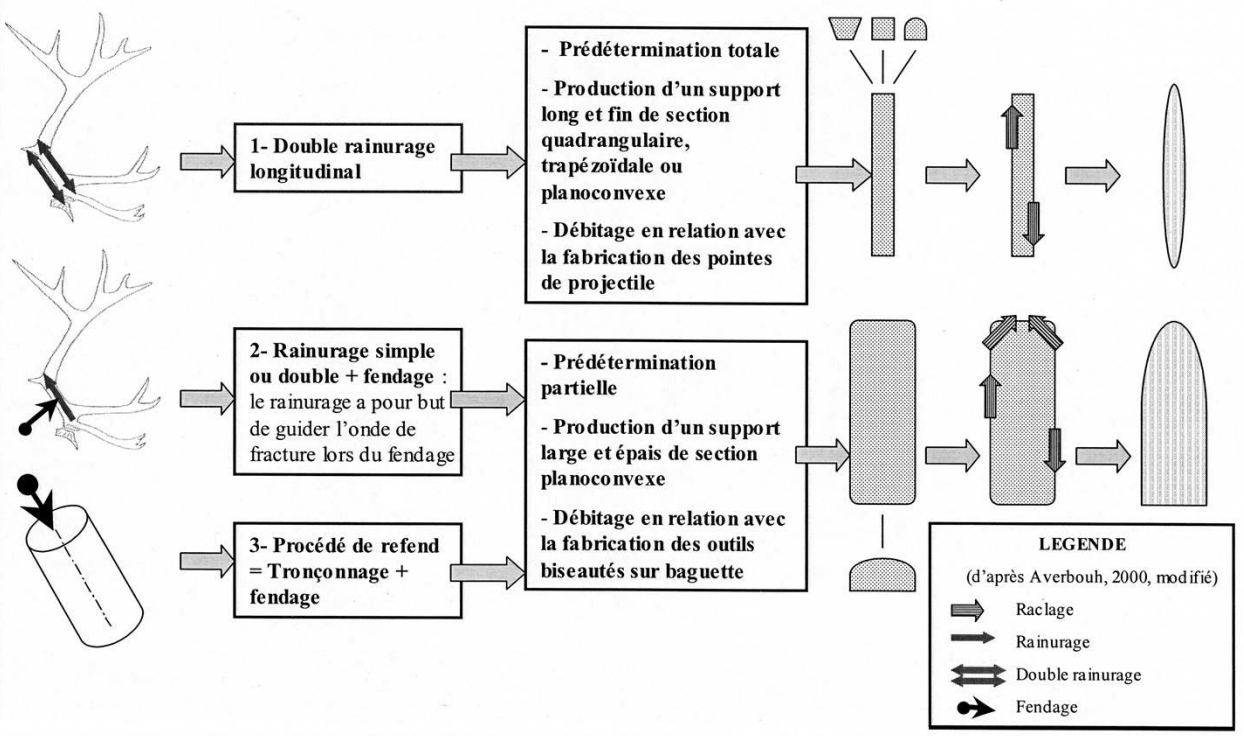

\section{1- Le procédé d'extraction de baguette par rainurage/fendage}

8 Sur certaines pièces, le double rainurage n'affecte qu'une faible longueur de la baguette à extraire, dans d'autres cas, seul un bord de la baguette a été en partie ou totalement délimité par rainurage (fig. 3). L'extraction est alors achevée par fendage. Cette utilisation de la fracturation contrôlée pour la production de supports allongés rappelle fortement le procédé aurignacien de refend. Cependant, dans le cas présent, le fendage est guidé partiellement par un rainurage simple ou double. Ce procédé d'extraction de baguette est très différent du double rainurage observé sur d'autres sites, dans les faciès ancien et moyen du Gravettien (La Gravette, Isturitz, Le Fourneau du Diable, l'Abri du Facteur...). Contrairement à ce dernier procédé, le rainurage n'a pas ici pour but de délimiter soigneusement la baguette sur toute sa longueur mais, davantage de contrôler les pans de fracture lors du fendage, en canalisant la propagation de l'onde de choc. 
Figure 3 - Extraction d'une baguette par le procédé de rainurage/fendage sur la face postérieure de la perche $A$ d'un bois de renne, (c) M.N.P.E, cliché Ph. Jugie, modifié.

Figure 3 - Extraction of a stick by slotting / splitting technique on the posterior face of a reindeer's wood (A perch), ( M.N.P.E, pict. Ph. Jugie, modified.
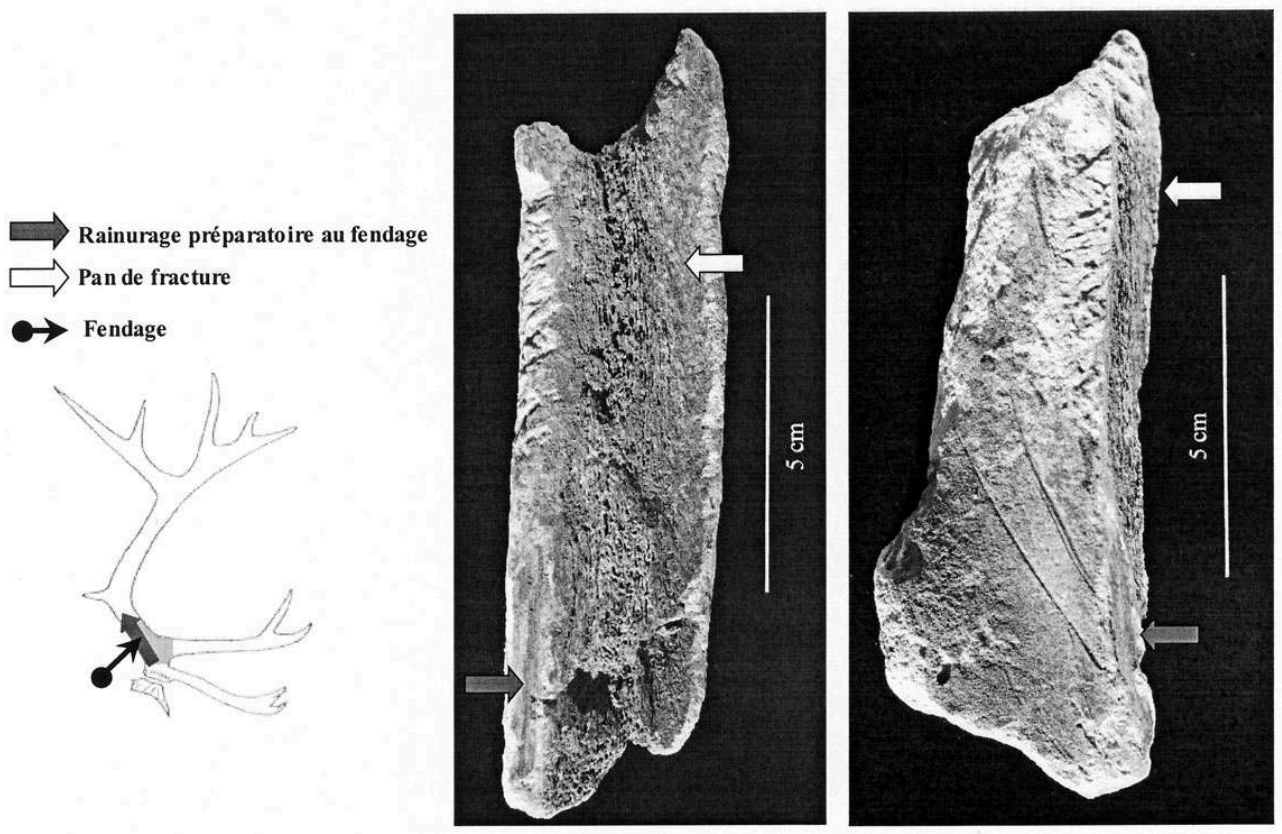

Un procédé similaire a été mis en évidence, par H. M. Bricker et N. David (1984) à l'Abri Pataud, au sein du niveau 3 appartenant lui aussi au Gravettien récent. Une courte rainure a été creusée au milieu d'une perche qui, selon ces auteurs, devait permettre l'insertion d'un coin facilitant le fendage du bois en deux:"Two pieces of antler (neither of which is illustrated) seem to indicate an alternative technique for dividing the shaft into convenient pieces. A burin has been used to cut a very short groove (40 to $50 \mathrm{~mm}$ long) somewhere near the middle of the shaft. The piece in its present form is split along the axis of the groove. Presumably the short groove provided a placed for the insertion of a wedge, which was then driven in to split the antler." (p.100).

\section{2 - Le procédé d'extraction de baguette par refend}

D'autres pièces, en revanche, témoignent de la seule utilisation du fendage à l'image du procédé aurignacien de refend. Ce dernier a été conduit sur des tronçons de perche ou d'andouiller. Il se caractérise sur les matrices et les baguettes, par des bords de détachement qui sont en réalité des pans de fracture.

\section{3 - Les objectifs du débitage par extraction de baguette à Laugerie- Haute Est}

11 Le double rainurage stricto sensu, permet la production de supports parfaitement prédéterminés et par-là même une plus grande normalisation de ces derniers. Cependant, il nécessite un temps de travail assez important.

12 A l'inverse, les procédés de refend et de rainurage/fendage permettent l'extraction rapide de supports allongés mais imposent en contrepartie, quelques contraintes. Ainsi, il existe un risque de fracture du support au cours du fendage. Pour pallier ce risque, il 
faut faire varier la largeur du produit linéairement avec la longueur : plus le support est long et plus il doit être large (Liolios 1999). Il n'est donc pas possible d'obtenir avec ces deux procédés, de fines et longues baguettes. Ce mode de débitage est plus rapide, mais il se fait aux dépens de la prédétermination morphologique des supports qui ne peuvent être vraiment normalisés. Si bien que le temps "gagné" au moment du débitage peut finalement être "perdu" au moment du façonnage si la forme de l'objet fini désiré est très éloignée de celle du support. Or, les seuls objets associés à ce mode de débitage à Laugerie-Haute Est sont des outils biseautés larges et épais (fig. 4), ne nécessitant pas d'étape de façonnage importante. En effet, ces derniers présentent une faible modification du support. Le produit est donc parfaitement adapté aux contraintes de ces procédés. Ce lien ne semble pas fortuit, il révèle plutôt que les Gravettiens, conscients des limites inhérentes à ce débitage faiblement prédéterminant, ont su en tirer avantage en les adaptant à leur objectif: celui de produire des supports larges et épais de section généralement planoconvexe et faiblement normalisés liés à la fabrication des outils biseautés (fig. 5).

Figure 4 - Outil biseauté sur baguette, @ M.N.P.E, cliché Ph. Jugie.

Figure 4 - Bevelled tool on stick (C M.N.P.E, pict. Ph. Jugie.

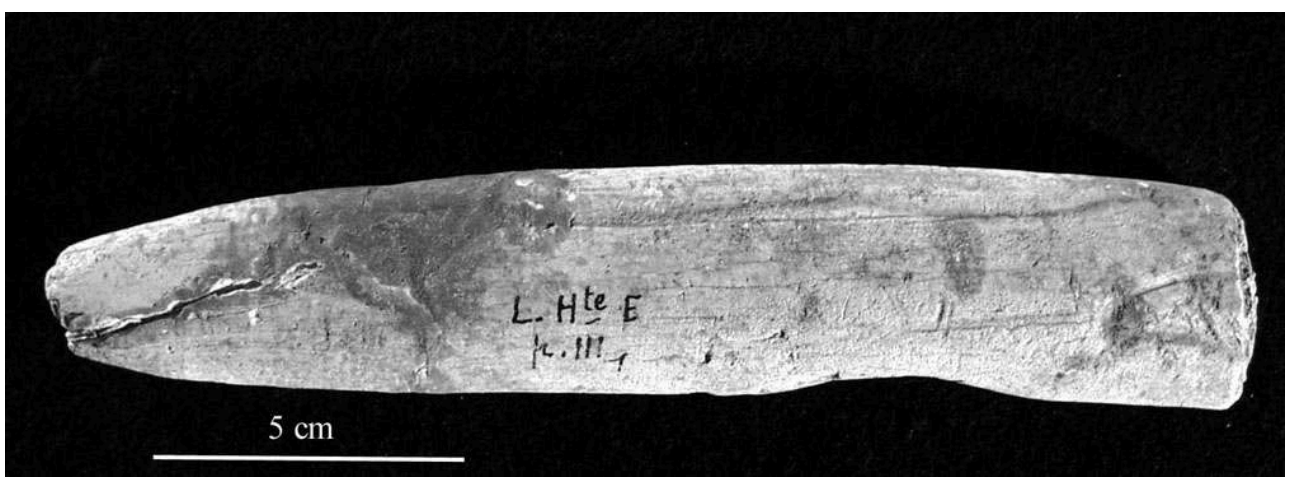


Figure 5 - Larges et épaisses baguettes obtenues par refend ou par rainurage/fendage, @ M.N.P.E, cliché $\mathrm{Ph}$. Jugie.

Figure 5 - Broad and thick sticks obtained by splitting and wedging or by slotting / splitting technique, (C) M.N.P.E, pict. Ph. Jugie.

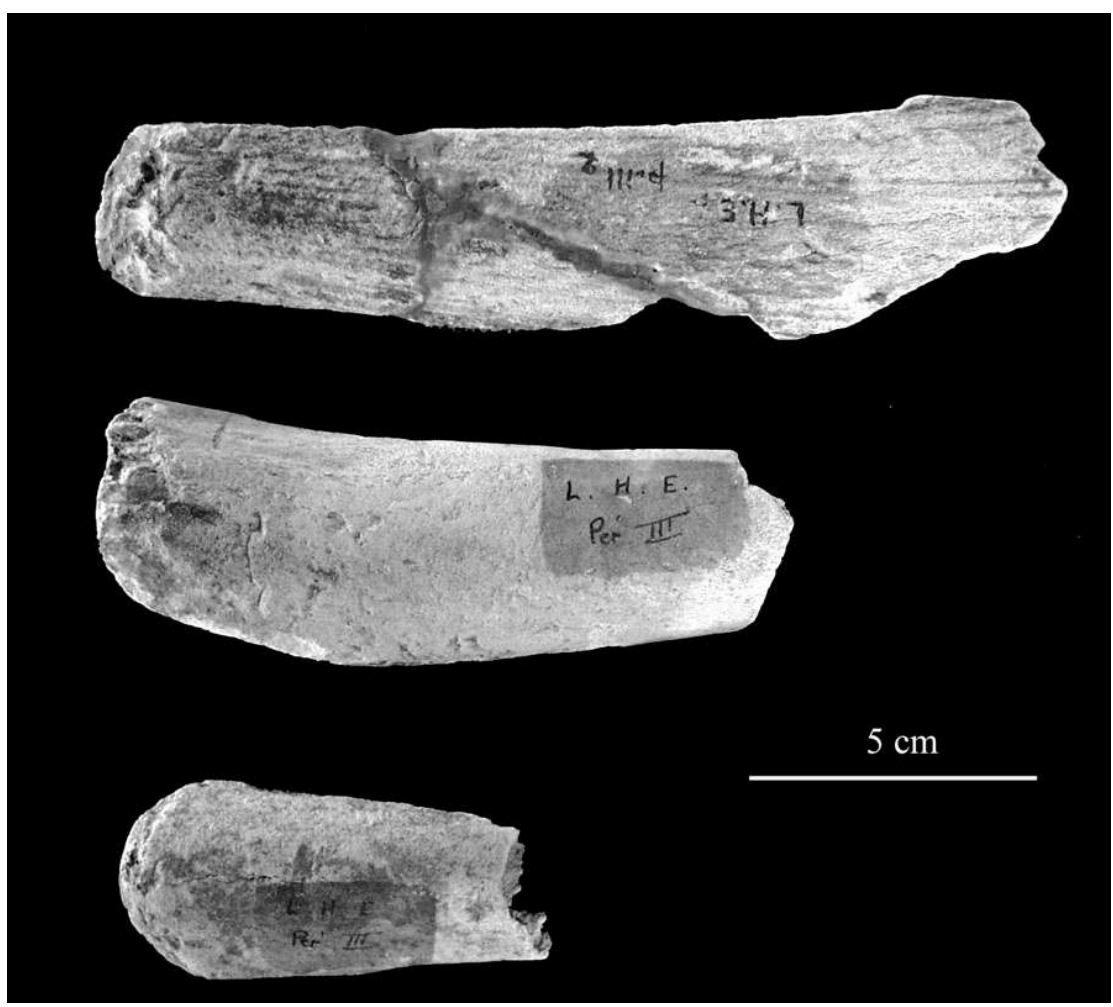

Ce procédé d'extraction en côtoie un second qui lui, permet d'obtenir des supports longs et étroits. Il s'agit du procédé de double rainurage longitudinal. Les baguettes ainsi obtenues sont de section quadrangulaire, trapézoidale ou planoconvexe suivant la localisation du débitage sur la perche et l'orientation du rainurage. Cette production est probablement à mettre en relation avec la fabrication des pointes de projectile. Cependant, la démonstration pèche par certains points. Les matrices témoignant de ce procédé présentent, en effet, des traces d'extraction de baguettes trop larges, pour correspondre parfaitement à la production de ce type d'objets. Néanmoins, certains supports bruts de débitage (fig. 6) ou en cours de façonnage (fig. 7) présentent des calibres et des morphologies tout à fait compatibles avec cette production. Aussi, nous émettons deux hypothèses :

- soit les matrices de débitage correspondantes se trouvent dans une zone non fouillée du gisement ;

- soit les pointes et les supports ont été apportés sur le site, leur production s'étant faite ailleurs. Cependant, s'agissant de fouilles anciennes, la maille de prélèvement n'était pas suffisamment fine pour permettre la récupération d'éventuels déchets de façonnage. Aussi, il n'est pas possible de dire si les pointes ont été apportées finies ou si leur façonnage s'est fait sur place. 
Figure 6 - Baguette brute de débitage extraite par double rainurage longitudinal, vue de la face inférieure, @ M.N.P.E, cliché Ph. Jugie.

Figure 6 - Sticks extracted by longitudinal groove and splinter technique, lower front view, (C) M.N.P.E, pict. Ph. Jugie.

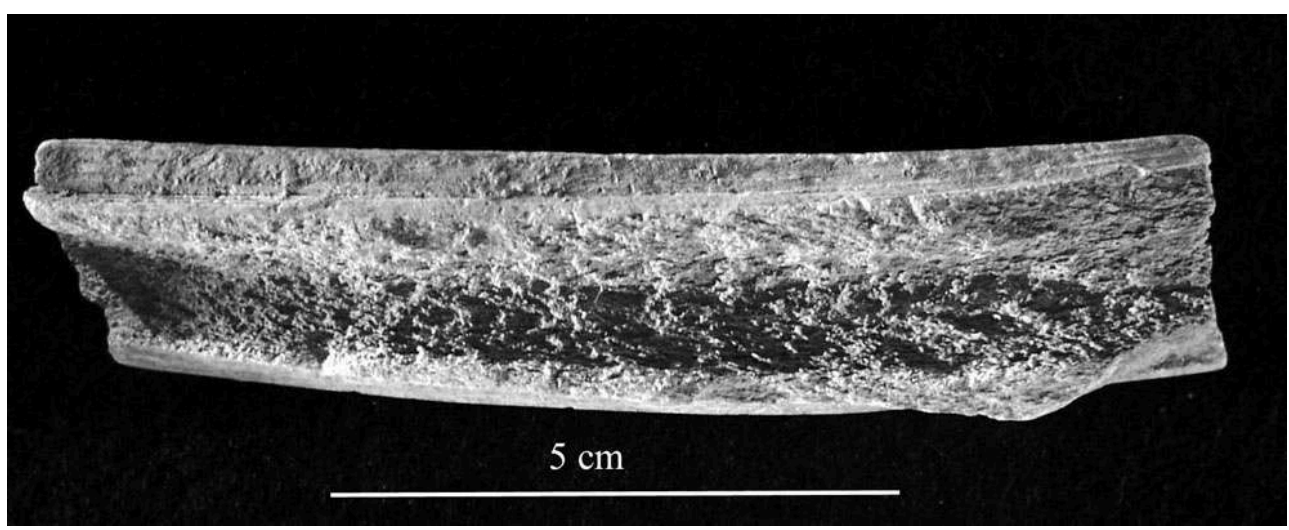

Figure 7 - Fines baguettes en cours de façonnage, de section quadrangulaire, @ M.N.P.E, cliché Ph. Jugie.

Figure 7 - Thin quadrangular section sticks been shaped, (C) M.N.P.E, pict. Ph. Jugie.

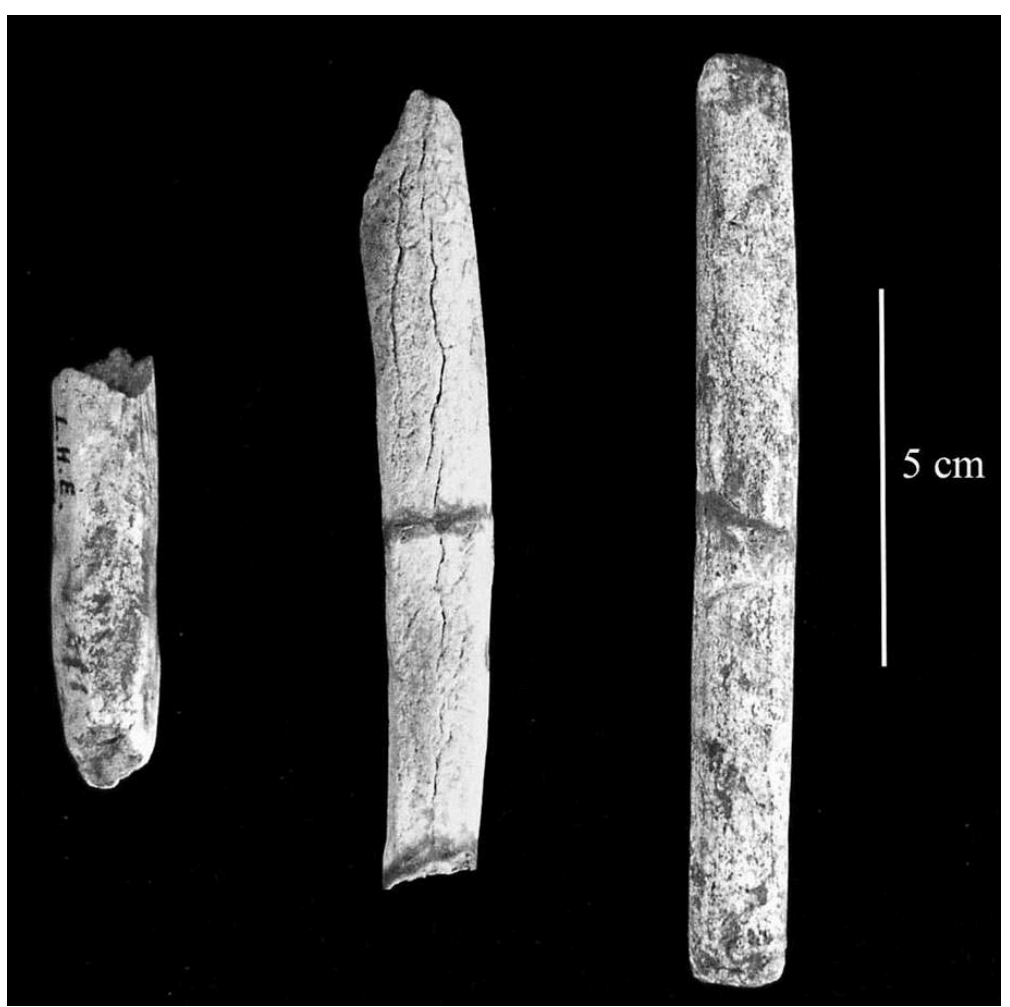

14 En définitive, il existe à Laugerie-Haute Est deux grandes modalités de débitage au sein du schéma de transformation par extraction, reposant sur l'emploi de trois procédés :

- le double rainurage longitudinal, dont l'objectif est la production de supports fins prédéterminés, de section quadrangulaire, trapézoïdale ou planoconvexe provenant principalement des faces latérales ou postérieure de la perche A ;

- le refend et le rainurage/fendage, dont l'objectif est la production de supports larges et épais, faiblement normalisés, de section planoconvexe et issus principalement des faces postérieure et antérieure de la perche A ou C. 

conséquent, par la morphologie des supports obtenus. Elles répondent à des objectifs différents adaptés aux contraintes et avantages de chacune.

L'utilisation conjointe du double rainurage, du rainurage/fendage et du refend, n'est pas selon nous, le reflet d'un "tâtonnement technique". En effet, le procédé de double rainurage est ici bien maîtrisé. Cette utilisation mixte semble donc répondre davantage à un besoin d'efficacité. Lorsque l'objectif était de produire des supports prédéterminés et hautement standardisés (pour la fabrication de pointes de projectile), les Gravettiens de Laugerie-Haute ont eu recours au procédé de double rainurage. En revanche, dans le cadre d'une production faiblement normalisée, relevant de l'outillage de fonds commun et principalement des outils biseautés, le refend ou le procédé de rainurage/fendage, rapides et efficaces, répondaient parfaitement à cet objectif.

\section{Un premier indice d'évolution diachronique dans le débitage des bois de cervidés au Gravettien ?}

17 Le procédé de refend était, jusqu'à présent, attesté uniquement à l'Aurignacien (Liolios 1999). Quant au procédé de rainurage/fendage, il ne semble pas connu à cette époque, en France (ibid.), en revanche, il est employé dans le débitage de l'ivoire sur des gisements allemands (Hahn 1995).

Cette persistance ou ce retour aux procédés de refend et de rainurage/fendage, dans les faciès récent et final $\mathrm{du}$ Gravettien est fort surprenante. En effet, les séries du Gravettien moyen que nous avons étudiées (Isturitz, l'Abri du Facteur, Le Flageolet) ne font pas état de ces procédés. Il eut paru moins surprenant que ces derniers soient utilisés dans les phases antérieures du Gravettien et non à la fin de ce dernier. Leur présence dans le Gravettien récent et final de Laugerie-Haute Est et Ouest est-elle le résultat d'une pérennisation et d'une amélioration d'une tradition technique aurignacienne (puisque ici le fendage est parfois associé au rainurage, ce qui n'est pas le cas à l'Aurignacien, en France) ? Ou bien, s'agit-il d'une réinvention témoignant d'une évolution diachronique dans le débitage des bois de cervidés faisant apparaître de nouveaux comportements techniques dans les phases récentes du Gravettien? Enfin, nous pourrions aussi considérer l'emploi de ces procédés à Laugerie-Haute comme le signe marqueur d'un groupe humain particulier et non comme celui d'une phase précise du Gravettien.

Dans l'hypothèse d'une pérennisation de ces procédés tout au long du Gravettien, comment se fait-il que nous n'en observions aucune trace dans les gisements des faciès moyens que nous avons étudiés et qui pourtant (mis à part Isturitz situé dans les Pyrénées-Atlantiques) proviennent tous de la même zone géographique (Dordogne). Si les séries appartenant au Gravettien moyen sont suffisamment nombreuses pour être représentatives, en revanche, celles du Gravettien ancien (La Gravette, Le Fourneau du Diable, Le Flageolet) ne le sont pas et les informations qu'elles ont livrées sur le sujet sont trop ténues pour que nous puissions vraiment nous prononcer. Bien que l'industrie osseuse de La Gravette et du Fourneau du Diable atteste de l'utilisation ponctuelle du double rainurage, cette constatation repose sur l'analyse de deux séries seulement, qui plus est, trop peu importantes pour être significatives, tandis que les données fournies par Le Flageolet sont très lacunaires et très difficiles à interpréter. Il 
est donc impossible d'affirmer que le double rainurage est le seul procédé d'extraction employé au Gravettien ancien. Qui plus est, à La Gravette, le double rainurage n'est attesté que sur l'os (par une matrice de débitage et un mésial de pointe). Quant au bois de cervidés, en raison de la disparité des traces de débitage longitudinal et du façonnage important des objets, le procédé d'extraction employé pour ces derniers est difficile à appréhender. Enfin, au Flageolet, l'industrie en bois de cervidés est rare et une seule pièce pourrait peut-être présenter les vestiges d'un double rainurage sur l'un de ses bords. En définitive, seul l'élargissement de notre étude à d'autres séries gravettiennes et la répétition de ces observations permettront de corroborer l'une ou l'autre de ces hypothèses.

\section{BIBLIOGRAPHIE}

AVERBOUH A. 2000 - Technologie de la matière osseuse travaillée et implications palethnologiques ; l'exemple des chaînes d'exploitation du bois de cervidé chez les Magdaléniens des Pyrénées. Thèse de Doctorat, Université de Paris I, 2 vol. , 500 p.

BILLAMBOZ A. 1979 - Les vestiges en bois de cervidés dans les gisements de l'époque Holocène. Essai d'identification de la ramure et de ses composantes pour l'étude technologique et l'interprétation palethnographique, Actes de la première réunion du groupe de travail sur l'industrie de l'os néolithique et de l'Âge des métaux, Aix-en-Provence, 1978, Paris, CNRS, 1979, p. 93-129.

RICKER H.-M. et DAVID N.-C. 1984 - Excavation of the abri Pataud, Les Eyzies(Dordogne), The Perigordian VI (level 3) Assemblage. American School of Prehistoric research bulletin, 34. Cambridge, Harvard University Press, Peabody Museum.

HAHN J. 1995. Les ivoires en Allemagne, débitage, façonnage et utilisation au Paléolithique supérieur. In Hahn J., Menu M., Taborin Y., Walter P., Widemann F. (éd.), Le travail et l'usage de l'ivoire au Paléolithique supérieur, Actes de la table ronde de Ravello, 29-31 mai 1992, Rome, p. 115-132.

LIOLIOS D. 1999 - Variabilité et caractéristique du travail des matières osseuses au début de l'Aurignacien, Approche technologique et économique. Thèse de Préhistoire et Technologie, Université de Paris X, $360 \mathrm{p}$.

PEYRONY D. et E. 1938 - Laugerie-Haute près des Eyzies (Dordogne). Archives de l'Institut de Paléontologie Humaine, Mémoires, 19, Masson, Paris, 86 p.

\section{NOTES}

1. Selon la terminologie développée par A. Averbouh (2000). 


\section{RÉSUMÉS}

La caractérisation des schémas opératoires de transformation des matières osseuses au Gravettien en France, n'en est pour l'instant qu'à ses balbutiements. L'étude de l'industrie osseuse des gisements de Laugerie-Haute Est et Ouest apporte de nouvelles informations sur le sujet. En effet, son étude nous a permis d'identifier des schémas opératoires inédits dans le débitage du bois de cervidés au Gravettien, mettant en jeu les procédés de refend - attestés jusqu'alors uniquement pour l'Aurignacien - et de rainurage/fendage.

The specification of bone and antler industry's production process for the Gravettian period in France is only in its infancy. The study of this industry in Laugerie Haute East and West put some new elements in the foreground concerning working of antler's industry. It shows especially unpublished production schemas for its debitage during the Gravettian period, involving splitting and wedging technique - attested until then for Aurignacian period only - and slotting/ splitting technique.

\section{INDEX}

Mots-clés : Gravettien, double rainurage, refend, rainurage/fendage

Keywords : Gravettian, groove and splinter technique, splitting and wedging technique, slotting/splitting technique

\section{AUTEUR}

\section{NEJMA GOUTAS}

UMR 7041-Archéologies et Sciences de l'Antiquité, Ethnologie préhistorique, 21, allée de l'Université, 92023 Nanterre cedex, France. 